\title{
Structural Features and Phylogenetic Implications of Two New Mitogenomes of Erythroneurine Leafhoppers (Hemiptera: Cicadellidae: Typhlocybinae)
}

\section{Xiaoxiao Chen}

Guizhou Normal University

Zhouwei Yuan

Guizhou Normal University

\section{Can Li}

Guiyang University

Christopher H Dietrich

University of Illinois

Yuehua Song ( $\square$ songyuehua@163.com)

Guizhou Normal University https://orcid.org/0000-0003-3567-3056

\section{Research article}

Keywords: Erythroneurine,mitochondrialgenome,nucleotidecomposition,divergencetime, Phylogeneticanalysis

Posted Date: September 30th, 2020

DOI: https://doi.org/10.21203/rs.3.rs-74836/v1

License: (c) (i) This work is licensed under a Creative Commons Attribution 4.0 International License.

Read Full License 


\section{Abstract}

Background: Complete mitochondrial genome sequences facilitate species identification and analyses of phylogenetic relationships. However, the available data are limited for the diverse and widespread insect familiy Cicadellidae. In this study, we sequenced complete mitochondrial genomes of two species of Erythroneurini.

Results: The mitogenomes of Empoascanara wengangensis and E. gracilis were 14,830 and 14,627 bp in length, respectively, and similar to other published leafhopper mitogenomes in terms of gene size, base composition, gene order, PCGs codon usage, and tRNA secondary structure. Most protein coding genes start with ATN and end with TAA or TAG. Two rRNA genes are highly conserved and encoded on the minority strand, and the AT content in $16 \mathrm{~S}$ is higher than that of $12 \mathrm{~S}$. The control regions in the genus Empoascanara are highly variable and contain various numbers of repeat sequences.

Conclusions: The mitogenomes of these two species closely resemble those of most other sequenced leafhoppers in various structural and compositional aspects. Phylogenetic analysis of 13 PCGs yielded a well-supported topology with most branches receiving maximum support and most relationships agreeing with those of other recent phylogenetic studies. Nucleotide diversity analysis show that nad4 and nad5 can be evaluated as potential DNA markers that define the Cicadellidae insect species. Like other studies, the main evolutionary event of leafhoppers occurred in the Tertiary, and its divergence time is estimated to be 10.03 122.48 Ma. This study confirms results of previous studies indicating that mitochondrial genome sequences are informative of leafhopper phylogeny.

\section{Full Text}

This preprint is available for download as a PDF.

\section{Figures}



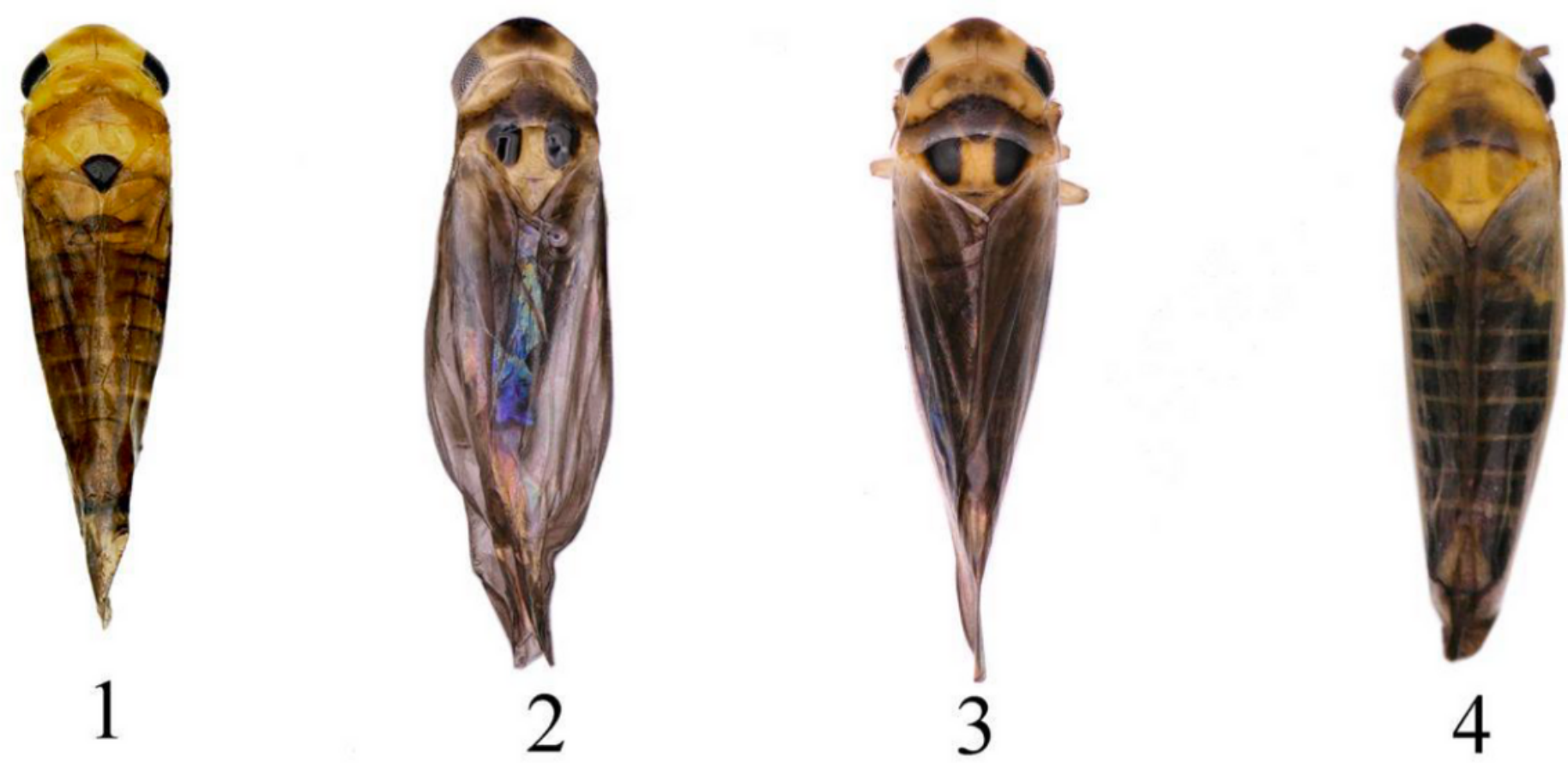

Figure 1

Four species of Empoascanara. 1 E. wengangensis Chen \& Song, 2020; 2 E. gracilis Dworakowska, 1992; 3 E. sipra Dworakowska, 1980; 4 E. dwalata Dworakowska, 1971.
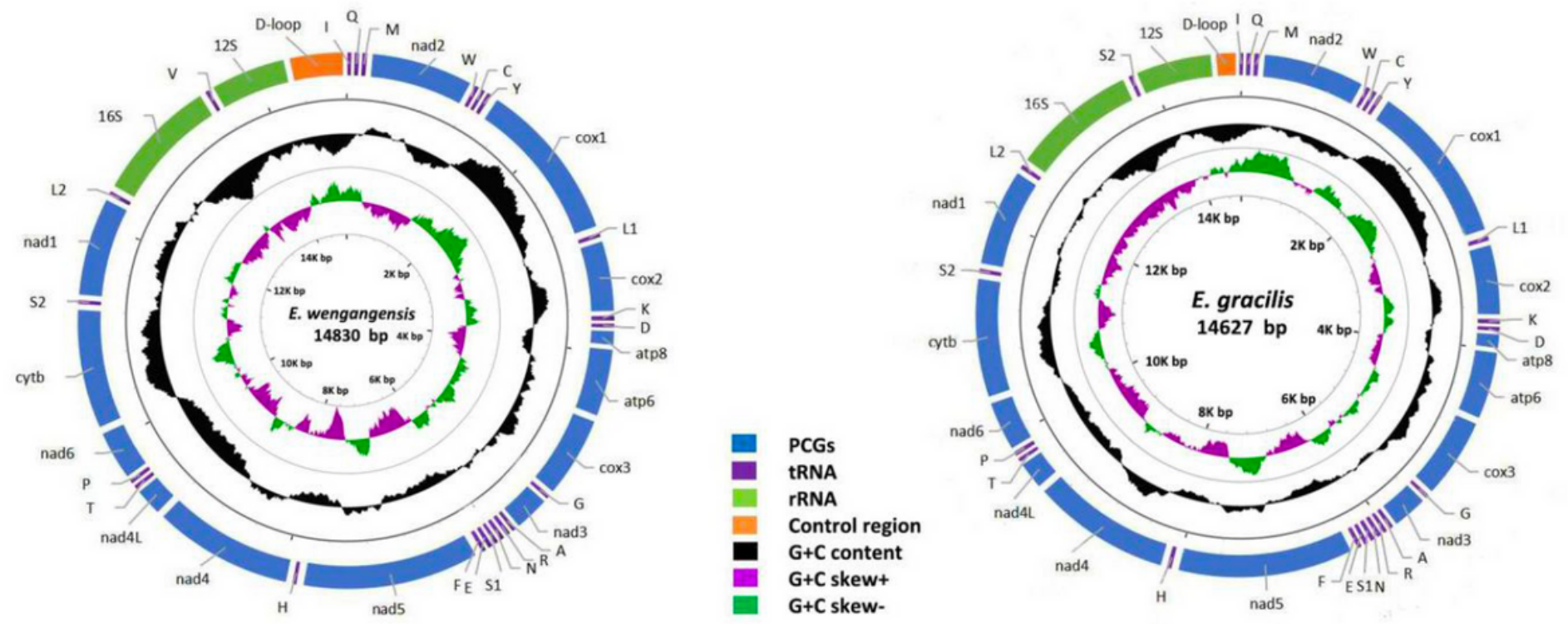

Figure 2

Circular map of the mitochondrial genome of E. wengangensis and E. gracilis. 


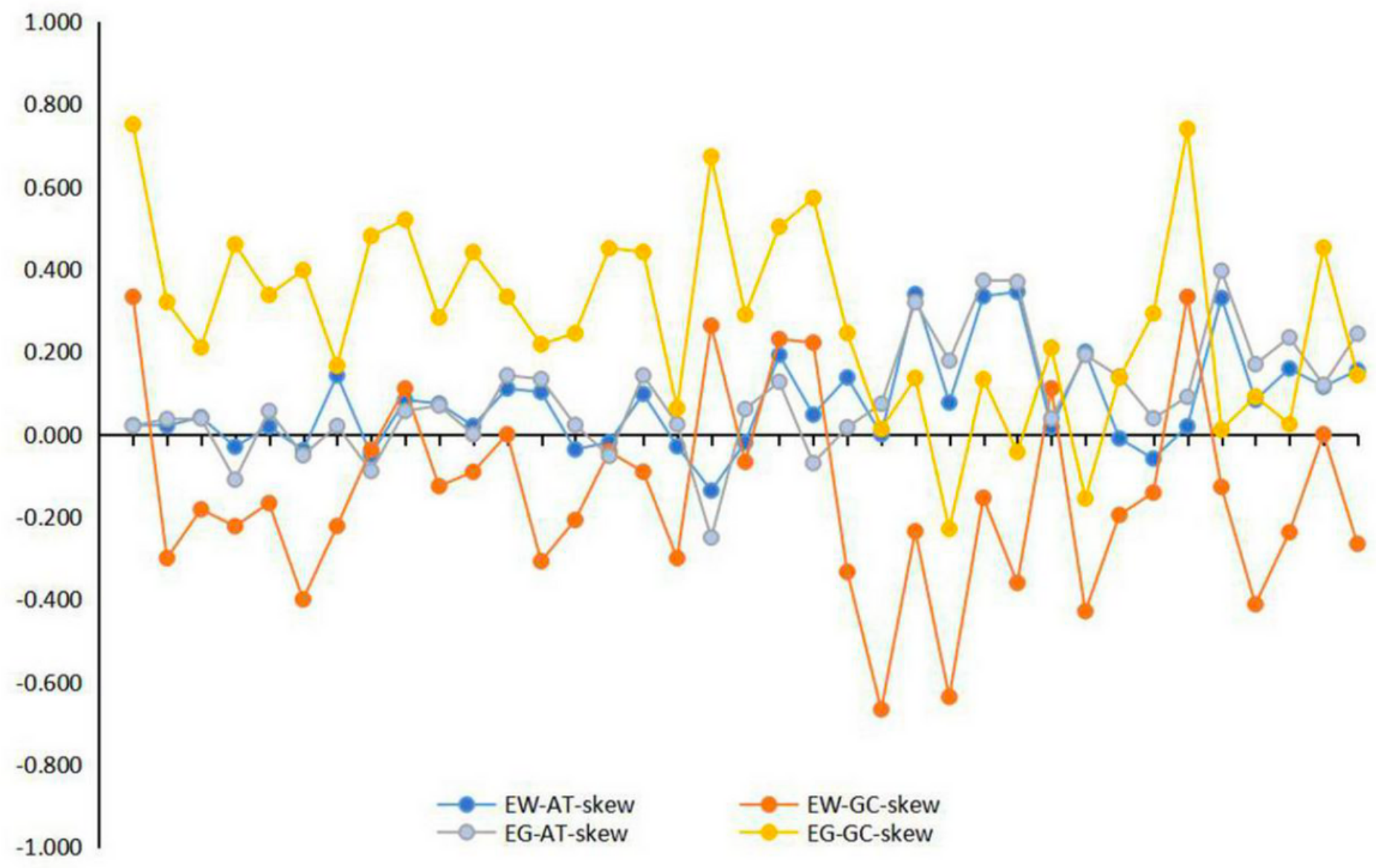

Figure 3

AT and GC skews calculated for the 37 mitochondrial genome of E. wengangensis and E. gracilis. Each point indicates an individual gene. 


\section{E. wengangensis}

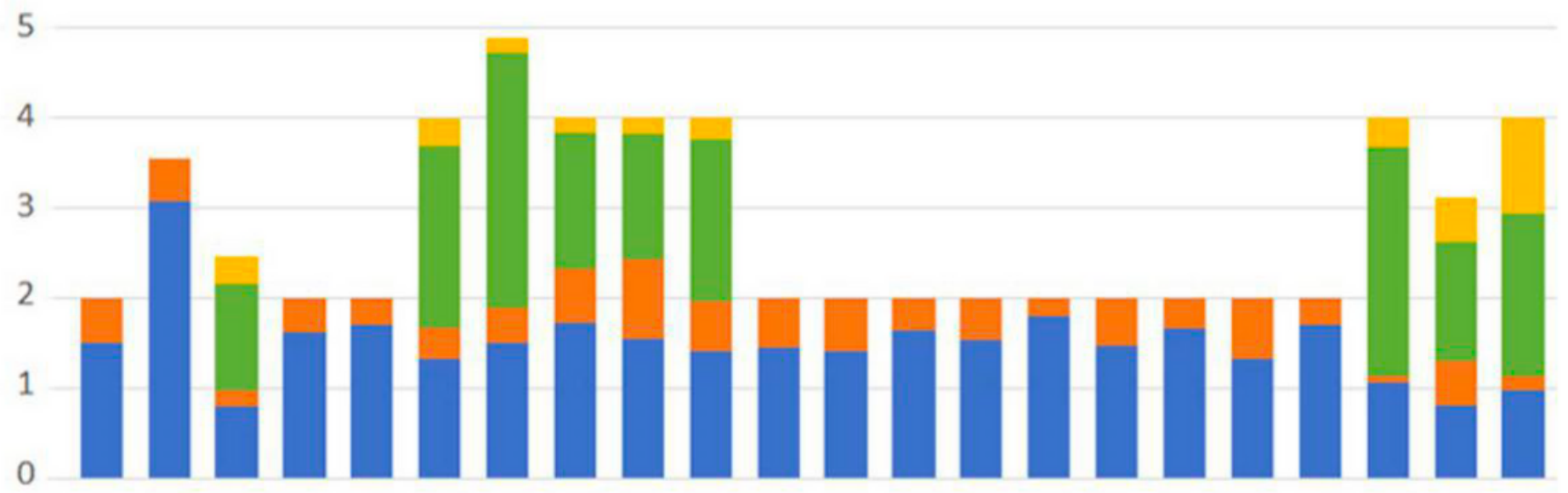

\section{E. gracilis}



\section{Figure 4}

Relative Synonymous Codon Usage (RSCU) of mitochondrial genomes for E. wengangensis and $\mathrm{E}$. gracilis. 



\section{Figure 5}

Inferred secondary structures of 22 tRNAs from E. wengangensis. Watson-Crick base pairings are illustrated by lines (-), whereas GU base pairings are illustrated by red dots. Structural elements in tRNA arms and loops are illustrated as for trnV. 
Empoascanara sipra

Empoascanara dwalata

\section{R1}

R2

\section{Empoascanara wengangensis}

\section{R1 R2}

\section{Figure 6}

Organization of the control region structure in the mitochondrial genomes of three Empoascanara species. R: repeat unit.

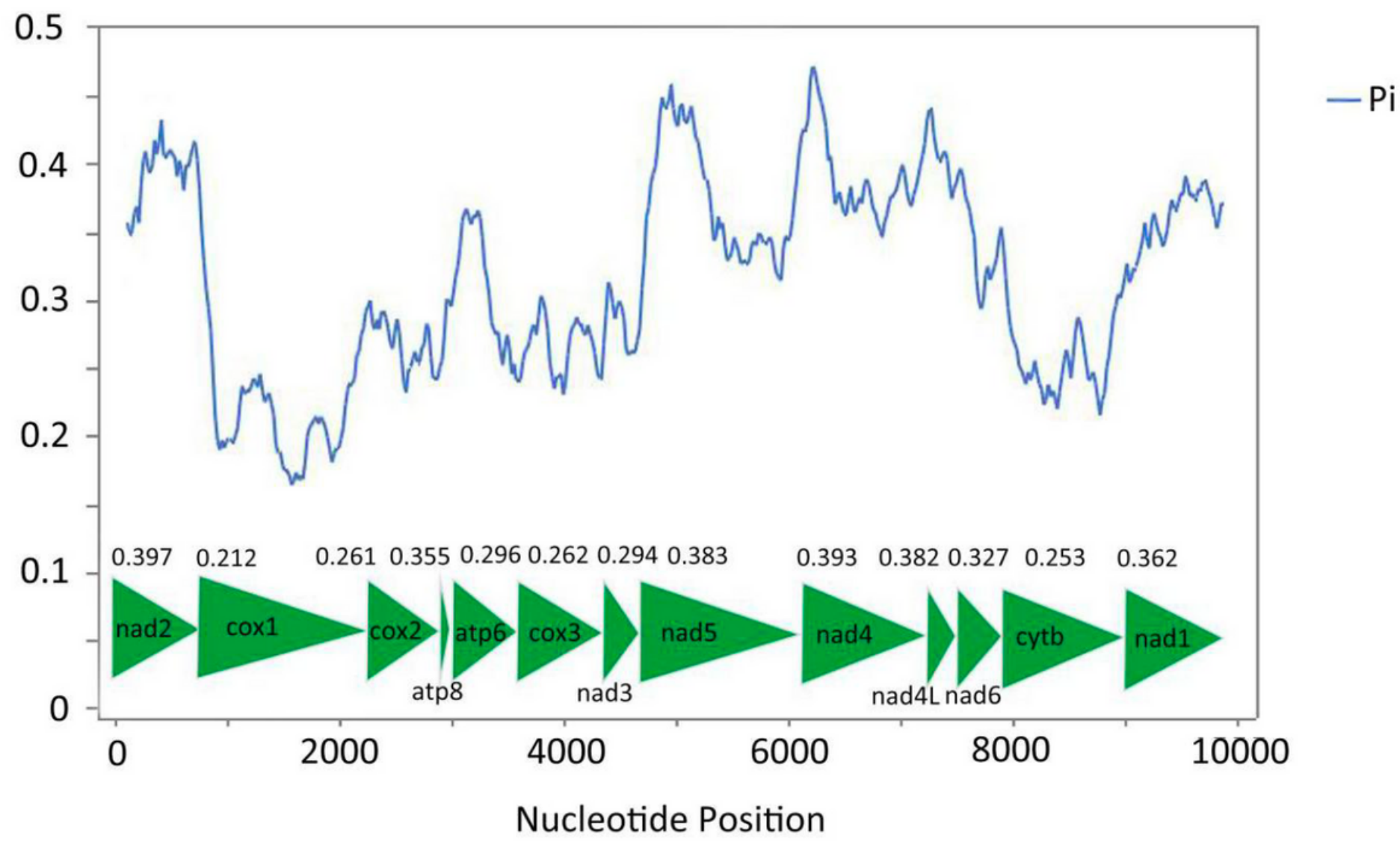

\section{Figure 7}

Nucleotide diversities and sliding window analysis of 13 PCGs of the 45 Cicadellidae species. Blue curve shows the value of nucleotide diversity $(\mathrm{Pi})$. Pi value of each PCG was shown above the arrows. 


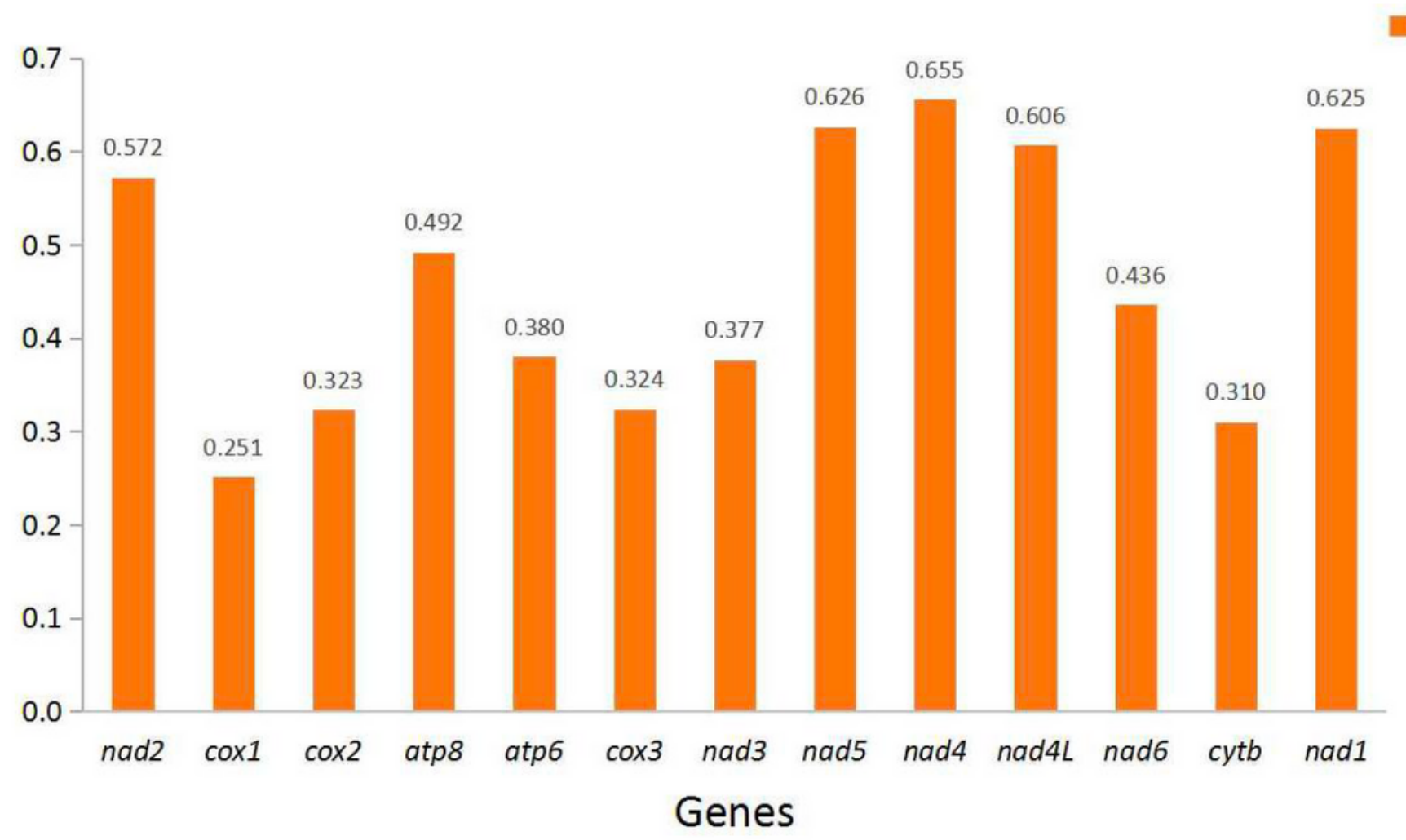

Figure 8

The ratio of non-synonymous (Ka) to synonymous (Ks) substitution rates of each 13 PCGs among 45 Cicadellidae species. 


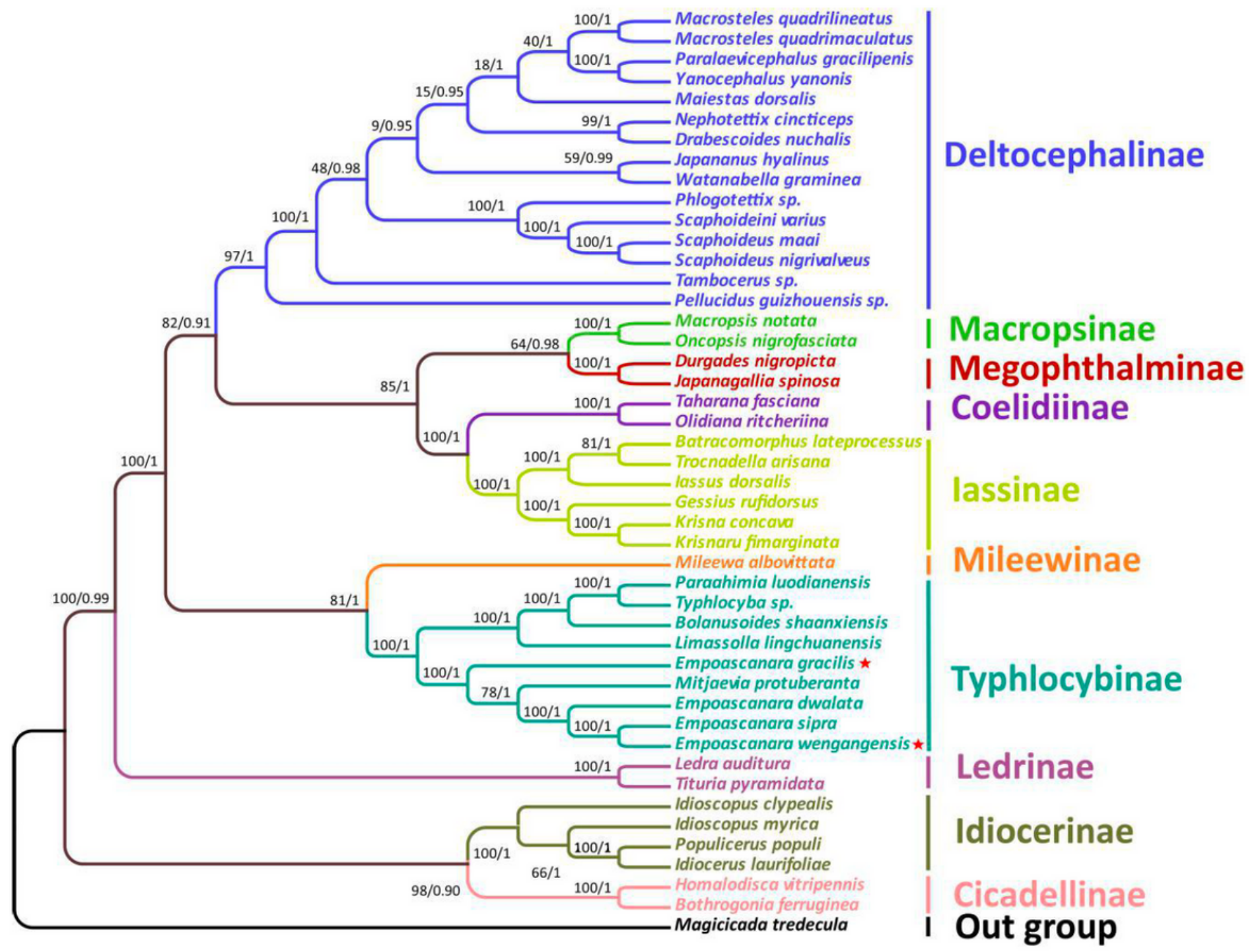

\section{Figure 9}

$\mathrm{ML}$ and BI Phylogenetic tree inferred from 13 PCGs of Cicadellidae. The first number at each node is bootstrap proportion (BP) of maximum likelihood (ML) analyses, and the second number is Bayesian (BI) posterior probability (PP). 


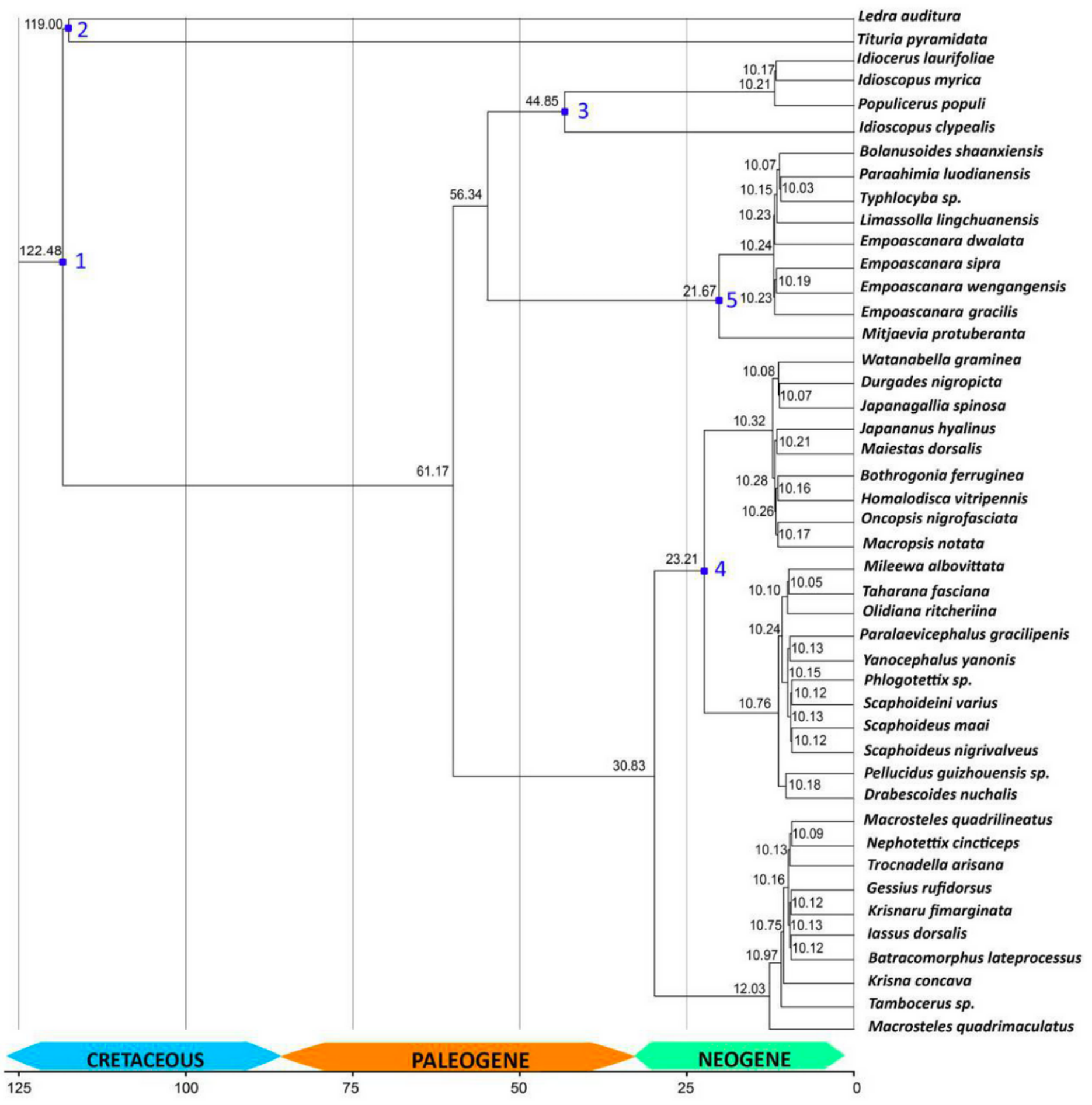

Figure 10

Dating tree showing divergence time estimates for major lineages of Cicadellidae. Numbered nodes marked in blue refer to fossil calibrations in Table 2.

\section{Supplementary Files}

This is a list of supplementary files associated with this preprint. Click to download. 
- SupplementaryMaterials.pdf

Page $11 / 11$ 